Microscopic Characters. I have made some very careful microscopic examinations of the crusts and secretion of impetigo contagiosa. In an early state, when the isolated points first make their appearance, there are present a few pus-cells perhaps, but chiefly a homogeneous blastema, with more or less fat, epithelial débris, and the like. In the more fully developed condition, the same elements are seen, but with a decided comparative preponderance of pus-cells. The scabs are composed of foreign substances (cotton, fibres, etc.), epithelial cells in different degrees of development, homogeneous plasma, pus-cells, fat, hairs, and parasites (animal and vegetable). I have never detected any sign of the presence of the acarus scabiei; but have noticed most perfect specimens of the steatozoon (acarus) folliculorum; and in several instances the spores and filaments of tricophyton, but in others not a trace; and, from all considerations, I believe the presence of the latter is an accidental phenomenon. It is worth mentioning, that the small hairs in the crusts are invaded and split up by the fungus-a state which, whatever may be said to the contrary, is, practically speaking, diagnostic of the presence of a parasite, provided the hair so split up be a well developed one. In the Transactions of the Pathological Society (vol. vi) may be found the brief description and figures of a supposed new fungus observed by Mr. Hutchinson in the course of " contagious porrigo". "Although", he says, "a vast number of cases were inspected, I was, however, successful in only two in finding anything beyond the usual constituents of purulent crusts." He adds : "Having failed to find, in so very large a proportion of the cases examined, anything at all similar to the bodies illustrated, I cannot, of course, suppose that the observation is of any value in relation to the pathology of the disease."

One word more about the umbilication, as it has been termed. In some cases it scarcely, perhaps, amounts to more than is entitled to be called depression; but the tendency to marked depression in the centre, as compared with the circumference, is certainly the characteristic of the spots of impetigo contagiosa ; and this feature is, as I have explained, dependent upon the peculiar behaviour of the secretory power of the disease. When thick crusts form, of course the aspect of umbilication is obliterated.

Causes. The disease exhibits no preference for sex. It attacks adults sometimes, but children most generally, and particularly those of the lower orders, of scrofulous habits - the fat, flabby, pale, thick-nosed, and light-complexioned ones. Want of cleanliness, light, and air, and wholesome food, help out its evolution. The disease not unfrequently follows in the wake of, or appears to be caused by, vaccination; at other times, it has no direct dependence whatever on this. In a person predisposed to the disease, it is not unlikely that any local irritant would call it forth into tangible existence.

[To be continued.]

\section{CASE OF EMBOLISM, FOLLOWING SYNCOPE FROM POST PARTUM HAMORRHAGE.}

By J. Shepherd Fletcher, M.D., Manchester. [Read before the Manchester Medical Society.]

[THe following notes of the case are furnished by A. Ransome, M.A., M.B.Cantab.]

Mrs. R., aged 36, had previously borne two children. She had phthisis sixteen years ago, from which she recovered, but has been delicate. Her brother died of phthisis, and her father of diabetes. She was con- fined February 12th, with a girl. The delivery was very rapid, and was shortly followed by profuse hæmorrhage, which produced slight fainting, lasting for some time. Her recovery was rapid. She was allowed to sit up on February 25th.

On March 1st, she rose at 10 A.M. She had suckled the baby frequently in the morning. She ate rather a smaller dinner than usual; but, in other respects, nothing unusual was noticed. At 2 P.M., she was sitting up. The nurse had just taken the baby from her, and turned away; on looking round the moment after, she saw Mrs. R. with her lower jaw dropped, pale, and making signs with her hands. The nurse gave her a tablespoonful of raw brandy, and then got her upon the sofa. Mrs. R. was just able to name her next neighbour, and made signs to send for her. After this, she could make no articulate sound.

When seen half an hour after the seizure, she was lying upon the sofa, pale and much agitated; her head was hot; her hands wet with perspiration; the pulse in right wrist (the only one then examined) 104, full, and jerking. She moved both hands, to express, as it seemed, something wrong about them. The face was somewhat drawn to the left side; and the tongue, when protruded, was pushed to the right side. All power of articulation was completely gone. Mustard was applied to the back of the neck, to the stomach, and to the calves of the legs. Two grains of calomel were given, and ammoniated tincture of valerian was ordered. In about an hour from the time of the attack, she seemed to recover partially, and again spoke easily and plainly; but in another half-hour she again became speechless, though conscious, and able to answer by signs.

At 5.30, Dr. Fletcher saw her, and found that, though the pulse was easily felt in the right wrist, yet it was scarcely perceptible in the left: it was equally feeble in the brachial artery, but was easily found in the axillary, subclavian, and carotid. She could move both hands, but was unable to grasp with the right. The pulse had now become very rapid and weak. Brandy and sal volatile, and then brandy and ammoniated tincture of valerian, were given every twenty minutes; and afterwards, five grains of sesquicarbonate of ammonia and half a grain of ammonio-tartrate of iron, in camphor julep, were given alternately with brandy and beef-tea every hour through the night. Blisters were applied behind the ears. Under this treatment, the pulse gradually improved slightly in strength, and was rather more easily felt in the left wrist; but it was still excessively feeble and small.

March 2nd. 8 A.m. She had dozed a little in the night. Her head was cool; the body was bathed in perspiration. The blisters had risen. The bowels were not open; the abdomen was distended with flatus. She had passed urine voluntarily every halfhour. The pulse was 96, stronger in both wrists, but still scarcely perceptible in the left. There was rather more strength in the right hand, but she was still unable to hold a pencil. An enema of turpentine and soap brought away a copious evacuation and much flatus. During the day, she gradually recovered power in the right side. The tongue, when protruded, was less completely pushed to the right side. The pulse also was diminished in frequency, but retained its force; and in the right wrist it had slightly increased in volume. The urine was pale, of specific gravity 1007 , not albuminous.

March 3rd. There was great improvement in her appearance. The skin was cool, not perspiring; pulse quieter in right wrist, stronger in left. She could hold a pencil in the right hand, and wrote several words on a slate without much difficulty.

March 5th. She articulated several words for the 469 
first time, as "baby, papa, beef-tea, good night." She wrote, that she felt more natural altogether, but complained of pain in the left shoulder, and of tingling in the left arm and leg. The bowels were regular; the pulse better.

March 6th. She seemed more nervous; had presentiments of impending evil. The pulse was softer and weaker. She wrote, that the sense of smell, which had been impaired, was now all right again.

From this time her progress was steady, but slow. Her speech was not perfect even so late as the end of May; but her strength had so far returned, that she was then able to go away from home.

The treatment by sesquicarbonate of ammonia and small doses of iron was continued throughout, with increase of dose, and the addition of a quantity of the tincture of nux vomica.

To the details of the case, as given above by $\mathrm{Mr}$. Ransome, I have nothing to add, except that, on the sixth day of July, she came by express train from the seaside in a great state of alarm, thinking another attack was impending, as she felt strange feelings of confusion in her head, with some unpleasant sensations in her right side. On careful examination, I could not detect any reason for increased fear, as in all respects she seemed better, except that the left pulse was still not equal to the right. I advised tepid shower-baths, and prescribed three grains of phosphate of zinc in pills three times a day. I saw her again in a month, when she expressed herself as quite well, and stated that the pills gave her immediate and most perfect relief from all her unpleasant feelings. Her speech up to about two months ago was slightly imperfect, and the left pulse below the right in strength.

The preceding case is remarkable for many circumstances, and is of considerable interest, inasmuch as the cause of the embolon, or clot, cannot have been such as is generally productive of this serious condition of things ; and, again, the result was fortunately altogether different to that which is the general rule in cases of embolism affecting the cerebral arterieswhich have been said to be almost always fatal.

The causes of embolism generally are known to be organic diseases of the left side of the heart, atheromatous degeneration of the large arterial trunks and inflammation of the veins; each one of these causes the development of fibrinous concretions, which adhere but slightly to the walls of the vessels or cavities in which they are contained, and are readily detached, when they are carried with the course of the circulation until they are arrested by some of the smaller vessels along which they are too large to pass.

The formation of fibrinous clots during syncope is an unusual cause of embolism, so far as I am acquainted with the literature of the subject ; and yet, looking at all the circumstances of this case, I can find no other reason for the formation of an embolon in the case just detailed. My own opinion as to this case is that, at the time of the hæmorrhage after labour, a small clot was formed, which, during the time of feeble circulation, probably contracted some adhesion to the walls of one of the heart's cavities-most probably the left ventricle-and when the circulation became more energetic, this was detached, and moving along the aorta, was arrested at the left carotid and the left subclavian arteries, into each of which it entered, fitting on the small portion of the aortic wall between them in the form of a saddle; from which spot a portion was probably detached to be carried into one of the cerebral arteries at the time that she for the second time became speechless and partially hemiplegic, and the second 470 portion into the lower end of the brachial when it stopped the pulse.

It will have been remarked that in the account given of the symptoms, the left side was the one in which the circulation was interrupted, whilst the right side was the one partially paralysed - this agreeing with the general rule as to cerebral paralysis.

Another remarkable feature in the case was, that as the pulse improved in the left wrist, the use of the right arm also returned; and these two symptoms seemed to move on together-to me very clearly telling of the same process of removal or absorption of the several clots. This is remarkably seen in the account of March 3rd: "Pulse quieter in right wrist; stronger in left; can hold a pencil in right hand."

On the 5th, she could write words ; and on the 6th, smell returned. It was a remarkable feature in the case that, although perfectly conscious, she could not for more than a week recollect the words she desired to write on her slate, and would very often use wrong words ; indeed, it was some weeks before she could always recollect the words she desired to use.

This lady had been for years subject to rheumatic pains; but she had never had any severe rheumatic attacks ; and, although I am quite aware that rheumatism has been found to be often associated with cases of embolism, I cannot suppose that this had any relation to this case-as I think it will be found that it is only in those cases of rheumatism giving rise to heart-disease that any connexion can be traced between the two conditions.

Certain conditions of the blood would undoubtedly give a disposition to the formation of clots, by deposit from the blood itself; and the condition of rheumatism, I apprehend, may be one of these.

In considering the question, as to whether or not an embolism can be formed in the blood by simple coagulation of a portion of its fibrine without the existence of any organic change in any part of the heart or blood-vessels-as I believe to have been the case here-we must remember that coagulation of the blood takes place much more readily with a slow and feeble circulation, and that after great loss of blood there is generally a relative excess of fibrine and a much quicker coagulation of the blood (Day), whether this loss occurs by blood-letting or by hæmorrhage.

Pregnancy, too, has its own particular condition of blood; viz., a low specific gravity, from an excess of water, fewer red corpuscles, and a relative excess of fibrine: all circumstances favouring the occurrence of embolism. Her anæmic habit is not an unimportant point in this case, and would aid in the development of an embolon.

The co-existence of all these favouring circumstances lends additional probability to my opinion, that the case I have narrated was one of the formation of an embolon by simple coagulation; and I think I am warranted in saying that such a pathological condition does occasionally occur. The prognosis in all such cases of embolism would be more favourable than in those arising from organic disease, from the fact of the clot being less firm than detached masses of old-standing effusions are; and referring to Dr. Richardson's experiments on the coagulation of the blood, we find that he could re-dissolve the clot of coagulated blood in serum alkalinified with ammonia. This I believe was done in the living subject in Mrs. R.'s case by the administration of ammonia; and $I$ am disposed to give a share of the credit due for the result of this case to Dr. Richardson, for the important additions he has made to our knowledge of the physiology of the blood, although I am quite 
aware that his conclusions as to the cause of the coagulation have been very ably contested.

The result of this case has been most satisfactory; for the long continued difficulty of articulation led to some little fear that a permanent difficulty of articulation might be the result, as it is said to be in all cases of recovery from embolism of the cerebral arteries.

Cases somewhat similar in character to the one I have detailed are, I am convinced, of much more frequent occurrence previous to and after confinement than are generally supposed; and are reported as, and believed to be simply cases of death from syncope. I have myself seen two cases, both of which proved fatal, occurring in the practice of other medical men; and which, I believe, were cases of embolism; but this disease not being thought of, was not detected, nor was any examination made after death. After great loss of blood, coupled with a feeble state of health, I think it will be well to keep the possibility of such an occurrence in our minds, and to direct such treatment as might tend to prevent it.

The treatment in this case is somewhat novel; as I find that most of the reported cases of embolism have been treated by calomel, croton oil, etc. Such treatment, carried to the extent of acting freely on the bowels, would, in this case, I think, have proved injurious; and in suggesting the use of the carbonate of ammonia, iron, and brandy, both $\mathrm{Mr}$. Ransome and myself were not influenced so much by the general condition of the system; but the special reason for preferring the carbonate of ammonia was founded upon Dr. B. W. Richardson's theory as to its action in maintaining the fluidity of the blood. Whatever may be thought of this theory, I am quite convinced that the line of treatment adopted in this case with such excellent results is the proper treatment for cases of embolism generally; and I should look with little favour upon the administration of calomel, croton oil, etc.

\section{CASE OF POISONING BY KIDNEY-BEANS.}

\section{By William Dale, Esq., Ilkeston.}

A FEW days since, $I$ was sent for to see a child five years old, who had been taken suddenly ill a short time previously, and was said to be dying. On my arrival at 8 P.M., I found him bedewed with cold perspiration; the breathing slow and sighing; the pulse at the wrist very rapid and almost imperceptible; the pupils widely dilated, and insensible to light. He could only be roused by speaking loudly, but appeared, when thus roused, to understand what was said. Pressure on the abdomen apparently gave pain, as the child shrank from it, and drew up his legs. The features also expressed suffering.

The history of the case was this. The child had always been remarkably healthy up to the time of this seizure. In the afternoon, he was playing with some kidney-beans. (These beans were some of last year's seeds, which a neighbour had thrown away because they were mildewed, and he thought them unlikely to germinate.) At tea-time, he asked his mother for some water. This almost immediately returned, and with it a quantity of half-digested beans. He then complained of pain in the bowels, and gradually became worse until I saw him. I immediately administered a dessert-spoonful of mustard in warm water; and, vomiting being produced, I ordered copious draughts of warm water, with an occasional teaspoonful of sweet oil. The vomit contained a large quantity of semi-digested kidneybeans. When the vomiting had somewhat abated, I ordered two teaspoonfuls of brandy to be administered occasionally, with a little water. The pulse now became a little stronger, and the breathing more natural; the pupils also began to act slightly, but the drowsiness still remained. He was then ordered to have two teaspoonfuls of castor oil. I also ordered a tablespoonful of strong coffee to be given every halfhour. The castor oil soon produced purging; and from that time amendment commenced. The pulse grew stronger; the breathing more natural; the pupils more active ; and the drowsiness less. In the morning, the only symptom remaining was a little tenderness at the epigastrium.

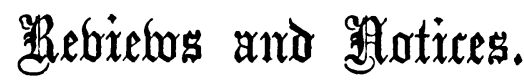

The Science and Art of Surgery: being a Treatise on Surgical Injuries, Diseases, and Operations. By John E. Erichsen, Professor of Surgery and of Clinical Surgery in University College, and Surgeon to University College Hospital. Fourth Edition, enlarged and carefully revised. Illustrated by 517 Engravings on Wood. Pp. 1280. London: 1864.

A System of Surgery. By James Miller, F.R.C.S.E. ; Surgeon in Ordinary to the Queen for Scotland; Professor of Surgery in the University of Edinburgh; etc. Pp. 1387. Edinburgh : 1864.

These two classical works on Surgery have appeared nearly simultaneously in London and Edinburgh; their authors being well known teachers of chirurgical knowledge in their respective localities. Mr. ERICHsen furnishes a fourth edition of his well known work. Mr. Mrller's book is, we believe, the result of the union into one volume, with the necessary emendations, of the two works which have hitherto been published under the titles of the Principles and the Practice of Surgery.

Of two such works, with the favourable antecedents belonging to them, there is little to be said by the reviewer; and any attempt to determine positively which is the better of the two would be, at the least, a difficult task in more senses than one. Yet, in their arrangement, and in some other matters, there is a marked difference between the two books.

Mr. Erichsen arranges his subjects under the three divisions of General Principles, Surgical Injuries, and Surgical Diseases; and in the latter two divisions, after first noticing each injury or disease in its general bearings, he describes the lesions affecting the various parts of the body. Pathological considerations, and the description of necessary operations, are with him incorporated with the description of the injury or disease to which they specially belong. An exception, however, occurs in the case of amputations, which have a chapter to themselves in the works of both authors-Mr. Erichsen placing at near the beginning of his book, while Mr. Miller places it at the end. Mr. Miller places his pathological remarks, and the general descriptions of such diseases as aneurism, fracture, dislocation, etc., in the first part of his book-that corresponding to his Principles of Surgery; while a regular arrangement of diseases and injuries is adopted in the remaining portion. Mr. Erichsen, considering that the diseases of the 